\title{
Metástasis única en el páncreas de melanoma de piel, resultados del tratamiento combinado con resección quirúrgica e inmunoterapia
}

\author{
Wilberth Méndez-Vivas ${ }^{1, *}$, Raúl Vázquez-Pelcastre ${ }^{2}$, Beatriz García-Robles ${ }^{4}$, \\ Marinée Torres-Aguilar ${ }^{5}$, Julio Ceballos-Zapata ${ }^{3}$ y Jorge García-Silva ${ }^{6}$
}

${ }^{1}$ Servicio de Oncología Quirúrgica; ${ }^{2}$ Servicio de Cirugía General; ${ }^{3}$ Servicio de Patología Clínica. Hospital Regional de Alta Especialidad de la Península de Yucatán, Mérida, Yucatán, México; ${ }^{4}$ Servicio de Radioterapia; ${ }^{5}$ Servicio de Oncología Médica. Hospital Galenia, Cancun Oncology Center, Cancún, Quintana Roo, México; ${ }^{6}$ Servicio de Endoscopia Intervencionista, Hospital Regional Mérida del Instituto de Seguridad y Servicios Sociales de los Trabajadores del Estado, Mérida, Yucatán, México

Recibido el 31 de agosto de 2016; aceptado el 25 de febrero de 2017

Disponible en Internet el 6 de noviembre de 2017

\section{PALABRAS CLAVES \\ Melanoma maligno \\ cutáneo; \\ Pancreatoduodenec- \\ tomia; \\ Inmunoterapia}

\section{KEY WORDS}

Cutaneous malignant melanoma;
Resumen Introducción: El melanoma metastásico al páncreas fue descrito por primera vez en 1931. Las metástasis pancreáticas se presentan con frecuencia en los pacientes con enfermeż dad difusa, la metástasis única es bastante rara; el papel de metastasectomía pancreática en pacientes con melanoma maligno no está definido en la actualidad debido a que existen muy pocos reportes disponibles. Caso clínico: Se revisa el caso de un paciente de sexo masculino de 54 años con historia de melanoma maligno nodular en muslo izquierdo EC IIIC en el que después de 11 meses de seguimiento se detecta una lesión metastásica única en cabeza de páncreas: Para mejorar nuestra comprensión de estas lesiones se describe un caso tratado con resección pancreática en nuestro hospital. Aquí, se discuten las características clínicas de su presentación, tratamiento y seguimiento junto con una revisión de la literatura. Discusión: La mayoría de los autores recomiendan la cirugía como el tratamiento de elección para las metástasis pancreáticas que son susceptibles de resección, ya que parece ser el único tratamiento capaz de prolongar la supervivencia, aunque no existen estudios grandes que demuestren totalmente este supuesto. Conclusión: Esta revisión sugiere que en un paciente con características tumorales favorables, la cirugía debe ser considerada como una opción viable de tratamiento y es necesario realizar estudios con mayor número de pacientes. (creativecommons.org/licenses/by-nc-nd/4.0/).

Abstract Introduction: Metastatic melanoma to the pancreas was first described in 1931. Pancreatic metastases occur frequently in patients with diffuse disease, solitary metastasis is quite rare; currently, the role of pancreatic metastasectomy in patients with malignant melanoma is not defined because there are very few reports available. Case report: The case of a male patient aged 54 with a history of nodular malignant melanoma left thigh EC IIIC, in

*E-mail para correspondencia: dr.wilberthmendez@hotmail.com (W. R. Méndez-Vivas) 
Pancreatoduodenec-

tomy;

Immunotherapy whom a single metastatic lesion is detected in pancreatic head after 11 months of follow-up, is revised to improve our understanding of these lesions, a treaty with pancreatic resection in our hospital case is described. Here, the clinical characteristics of presentation, treatment and monitoring are discussed along with a review of the literature. Discussion: Most authors recommend surgery as the treatment of choice for pancreatic metastases that are amenable to resection, as it seems to be the only treatment able to prolong survival, although there are no large studies that fully demonstrate this course. Conclusion: This review suggests that in a patient with favorable tumor characteristics, surgery should be considered a viable treatment option and it is necessary to conduct studies with more patients.

\section{CASO CLÍNICO}

Se revisa el caso de un paciente de sexo masculino de 54 años caucásico, originario de Alemania y residente en México desde hace 14 años, vegetariano y sin antecedentes de enfermedades crónicas, tiene historia de melanoma maligno nodular ulcerado de piel en muslo izquierdo con una profundidad Breslow de $6.5 \mathrm{~mm}$, nivel V de Clark, tres mitosis por $\mathrm{mm}$, de dos años de evolución, tratado con resección amplia de la lesión más disección ganglionar inguinal con siete ganglios positivos; se cataloga como EC IIIC por pN3, posteriormente recibe tratamiento adyuvante con RT50 Gy en 20 fracciones en acelerador lineal a región inguinal con fotones $6 \mathrm{MV}$ técnica de intensidad modulada en arco dinámico (VMAT). Se realizó tomografía por emisión de positrones- tomografía computarizada (PET-TC) de seguimiento dos meses después del evento quirúrgico, con reporte de aumento de SUV máximo de 5.8 en zona heterogénea de tejidos blandos además de prominencia del canal inguinal derecho con SUV máx de 4 en relación con inflamación (Fig. 1). Se consideró candidato a adyuvancia con interferón alfa $2 \mathrm{~b}$ a dosis de 10 mu de lunes a viernes durante cuatro semanas y posteriormente dosis de 10 mu tres veces por semana durante 12 meses. Después de 11 meses de seguimiento asintomático, en una TC abdominal de control se documenta dilatación del conducto pancreático principal condicionado por pequeña masa tumoral, con lipasa y amilasa en más de 2,000 UI sin hiperbilirrubinemia (Fig. 2). Se realiza otra PET-TC y se documenta un tumor sólido en cabeza de páncreas con SUV de 8; por ultrasonido endos- cópico se demuestra una lesión en la cabeza del páncreas cercana al cuello de 10 por $7 \mathrm{~mm}$, se realiza biopsia por aspiración con aguja fina (BAAF) y se determina tumor neuroendocrino maligno (Fig. 3), con marcadores tumorales negativos ACE y CA 19-9, sin síndrome carcinoide con cro mogranina A y 5-HIAA negativos. Se llevan a cabo estudios de extensión, con resultados negativos; se realiza gammagrafía con octreótido del páncreas, que se reporta positivo solo en cabeza de páncreas, se cataloga como segundo primario. Se realiza pancreatoduodenectomía sin preser vación pilórica, sin complicaciones. Durante la cirugía se halla un tumor en el cuello del páncreas, se palpa sólido, de aproximadamente $1.5 \mathrm{~cm}$, que condiciona la dilatación importante del conducto de Wirsung (Fig. 4), los ganglios macroscópicamente son negativos. Es egresado nueve días después del evento quirúrgico por mejoría. En el informe de patología se determina melanoma metastásico de 1.2 x $0.9 \times 0.9 \mathrm{~cm}$ localizado en el cuello del páncreas con márgenes negativos, necrosis tumoral, invasión linfática, vascular y perineural presentes (Fig. 5), y todos los ganglios negativos a metástasis, se corrobora el diagnóstico con estudios de inmunohistoquímica, proteína S 100, HMB 45, MART-1 y torosinasa positivos, el paciente evoluciona satisfactoriamente con dos meses de seguimiento asinto mático y sin evidencia de recaída en otros sitios, se realiza determinación de BRAF V600, siendo negativo, por lo que se decide iniciar tratamiento con ipilimumab. Doce meses después de completar el tratamiento, el paciente se en cuentra en vigilancia y asintomático sin evidencia de progresión de la enfermedad.
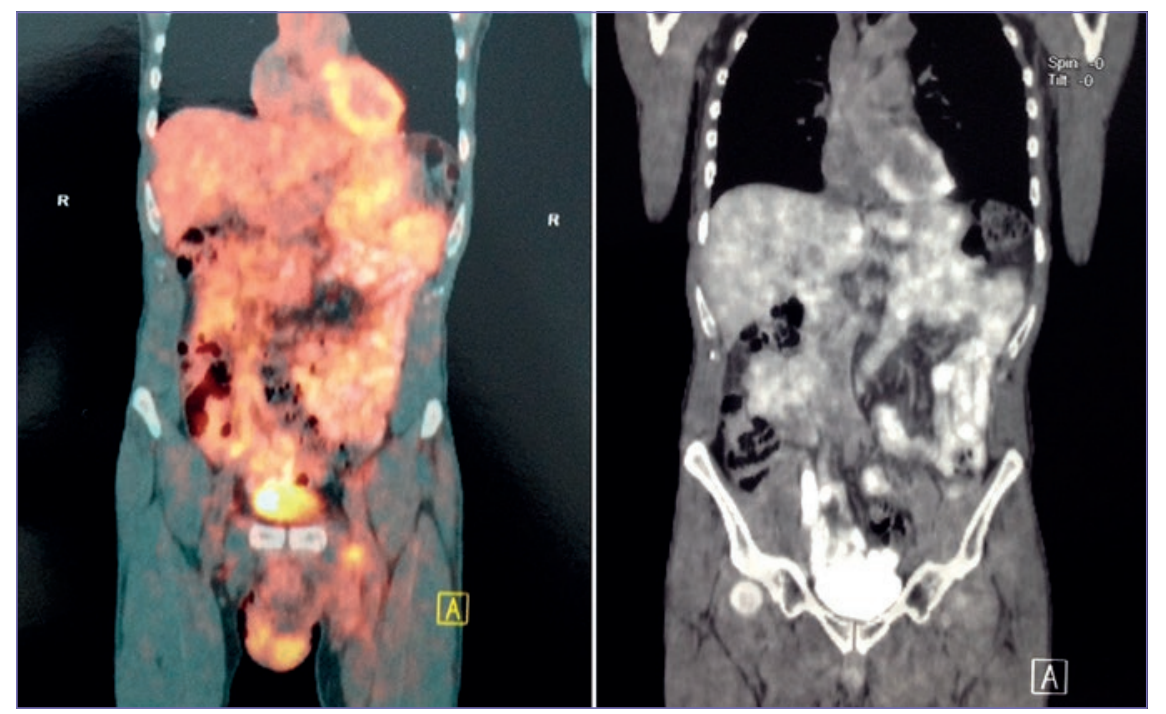

Figura 1. PET-TC ${ }^{18} \mathrm{~F}$-FDG dos meses después del tratamiento inicial con reporte de aumento de SUV máximo de 5.8 en zona heterogénea de tejidos blandos además de prominencia del canal inguinal derecho con SUV máx de 4 en relación con inflamación, no se aprecian más lesiones a distancia. 


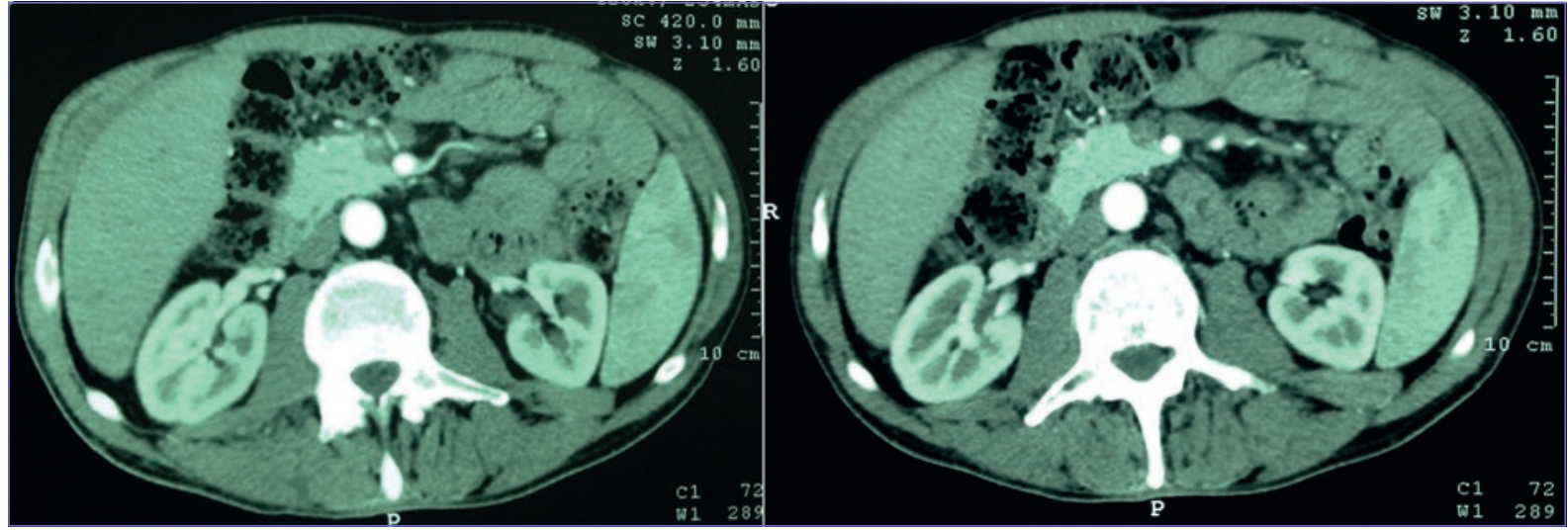

Figura 2. Tomografía helicoidal de abdomen con doble contraste 11 meses después del tratamiento inicial. Se aprecia dilatación del conducto pancreático principal condicionado por pequeña masa tumoral, se sospecha de segundo primario.

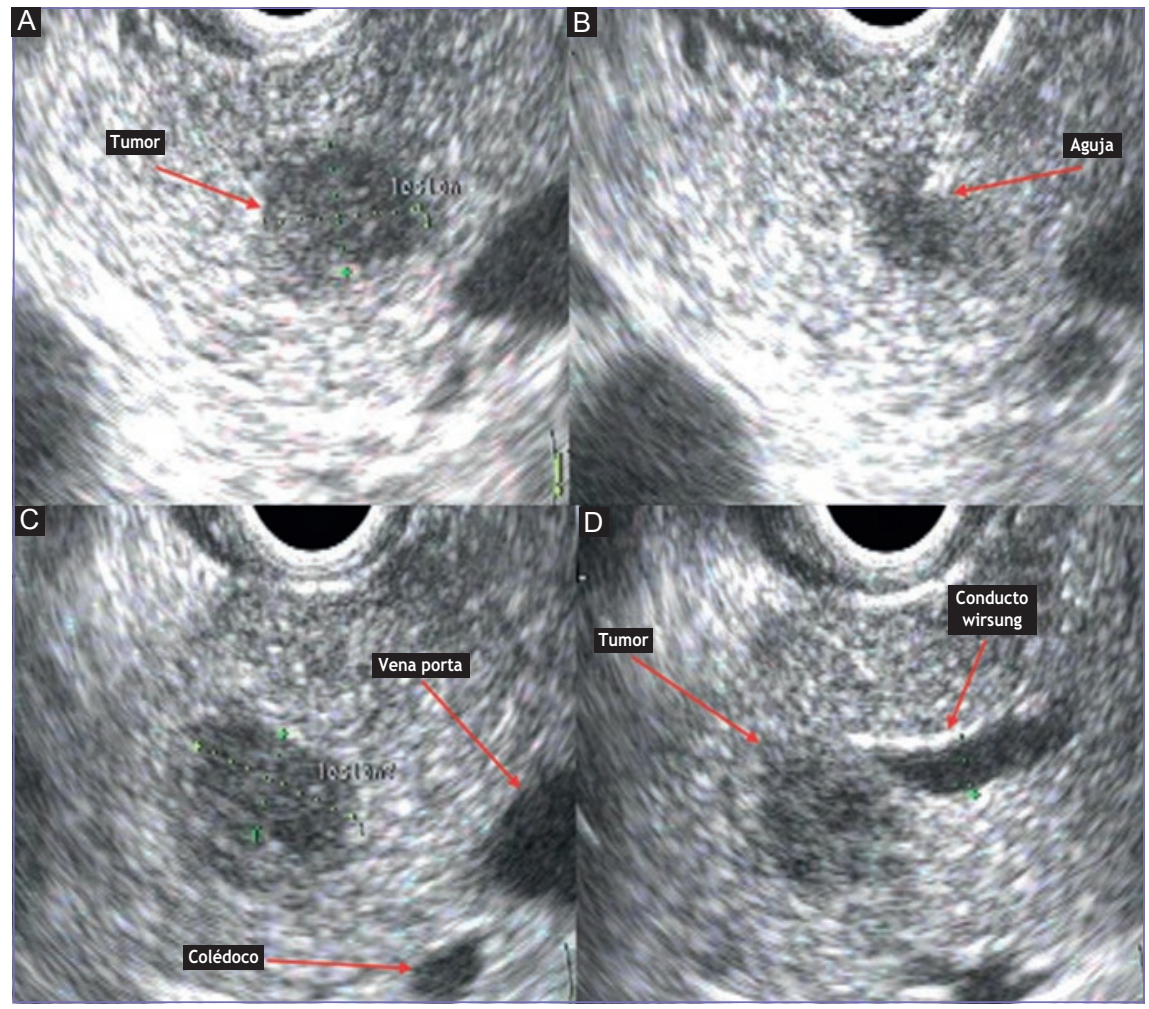

Figura 3. Ultrasonido endoscópico y biopsia por aspiración con aguja fina. En la imagen A se aprecia el tumor, en la B el momento de la toma de la biopsia, en el $\mathrm{C}$ la relación con la vena porta y el colédoco, y en $\mathrm{D}$ el tumor con la obstrucción del conducto pancreático principal que se muestra dilatado.

\section{DISCUSIÓN}

La metástasis aislada de otro tumor primario en el páncreas es muy rara $(<2 \%)^{1,2}$ y augura un mal pronóstico, con una supervivencia a los cinco años de menos del $10 \%$ y una mediana de supervivencia de 6-9 meses $^{3}$. A pesar de esto, varios estudios retrospectivos han sugerido un aumento de la supervivencia después de la resección completa de las metástasis pancreáticas en un solo sitio, generando un gran interés en este enfoque ${ }^{4-6}$. Hay, sin embargo, muy poca literatura sobre la resección pancreática para el melanoma metastásico. Las metástasis pancreáticas ocurren solo en el $2 \%$ de los casos de enfermedad metastásica resecable ${ }^{7}$, los primarios comunes que hacen metástasis en el páncreas son de mama, pulmón, renales y de colon y con menor frecuencia, el melanoma y el sarcoma ${ }^{6}$. El melanoma metastásico al páncreas fue descrito por primera vez en 1931; las metástasis pancreáticas se presentan con frecuencia en los pacientes con enfermedad difusa, la metástasis única es bastante rara y se ha descrito mayormente en el melanoma ocular primario ${ }^{2}$. Por otro lado, el melanoma metastásico tiene un pronóstico desfavorable, la supervivencia a cinco años de los pacientes con una metástasis única es del $12 \%$ (mediana de 11 meses), mientras que la supervivencia a los cinco años con múltiples metástasis es del 0\% (mediana de supervivencia, cuatro meses). Después de una resección completa de la metástasis, la supervivencia a los cinco años aumenta de forma significativa al $18 \%$, con una superviven- 


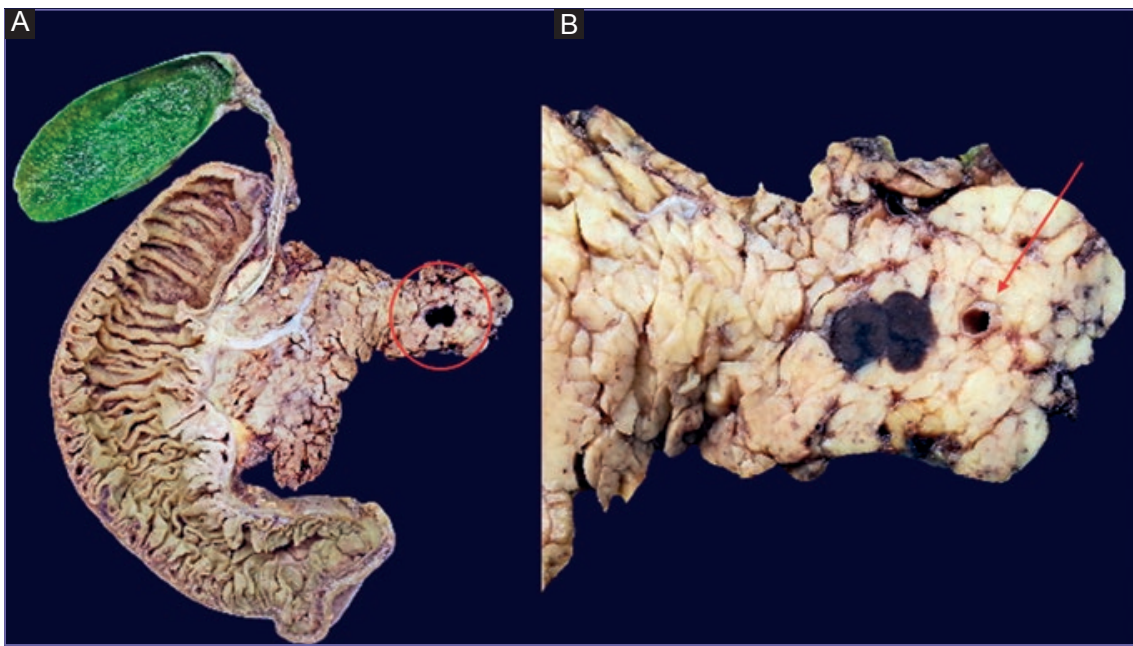

Figura 4. Imagen macroscópica de la pieza, en el cuello del páncreas (A) se identifica una lesión (círculo rojo) que mide $1.2 \times 0.9 \times 0.9 \mathrm{~cm}$, es de aspecto neoplásico maligno, ovoide, de bordes lobulados, empujantes, de consistencia dura y de color negro. Se encuentra localizada a $1.5 \mathrm{~cm}$ del margen quirúrgico pancreático, a la derecha (B) se aprecia una ampliación del tumor, se aprecia además el conducto pancreático principal, dilatado (flecha roja).
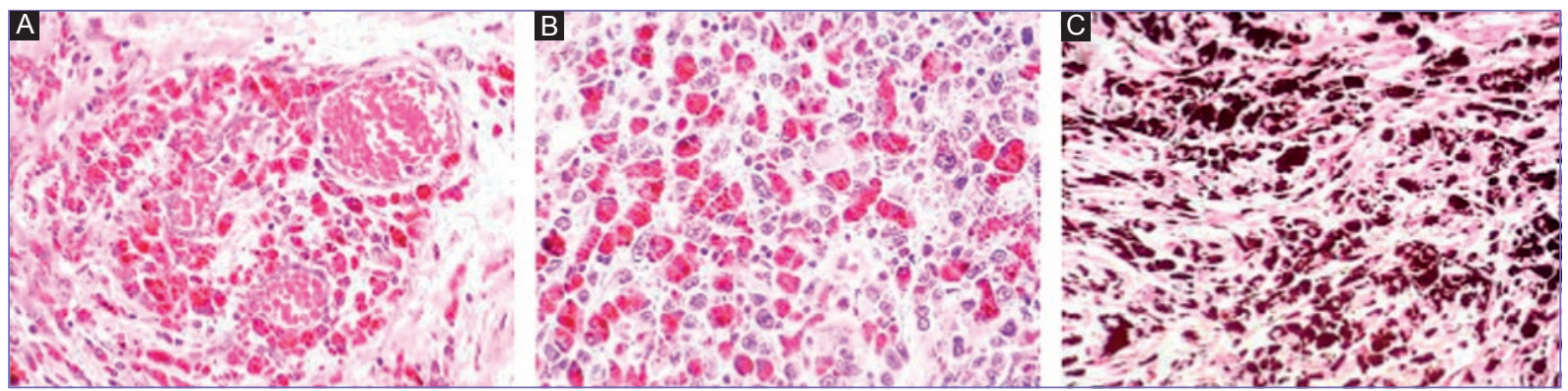

Figura 5. Microfotografías, el tumor se caracteriza por la proliferación de células cúbicas a poligonales con amplio citoplasma eosinófilo denso (A), muchas de ellas con pigmento café oscuro (B), que con tinción de Fontana-Masson (C) adquiere un tinte negro, melanina. Los núcleos tienen atipia celular marcada, la mayoría de ellos vesiculados con nucléolos grandes, eosinófilos. Se identifican células neoplásicas dentro de vasos y alrededor de tractos neurales (A).

cia media de 15 meses. La supervivencia es dependiente del sitio de metástasis a distancia; los pacientes con afectación visceral tienen resultados menos favorables que aquellos con afectación de los tejidos blandos o recidiva ganglionar distante ${ }^{8}$. En un estudio retrospectivo de 49 casos realizado en el Hospital John Hopkins en pacientes con metástasis a páncreas, los tres pacientes con melanoma metastásico tuvieron peor pronóstico ${ }^{9}$. El papel de metastasectomía pancreática en pacientes con melanoma maligno no está definido en la actualidad debido a que existen muy pocos estudios disponibles ${ }^{4,10}$. Tradicionalmente, la metastasectomía se consideró inútil debido a un mal pronóstico asociado con la enfermedad muy extendida. Sin embargo, algunos pacientes con enfermedad metastásica limitada logran sobrevivir por un tiempo razonable después de la cirugía ${ }^{2}$ e incluso existen informes de curación ${ }^{11}$. Esto, junto con una mejora en el riesgo operativo asociado con el procedimiento demostrado por muchos grupos, ha dado lugar a un interés renovado en el tratamiento quirúrgico del melanoma metastásico. La mayoría de los autores recomiendan la cirugía como el tratamiento de elección para las metástasis pancreáticas que son susceptibles de resección, ya que parece ser el único tratamiento capaz de prolongar la supervivencia ${ }^{9,12}$. También parecen tener un índice de resecabilidad más alto en comparación con el adenocarcinoma de páncreas; esto se debe a que los límites de las lesiones metastásicas tienden a estar mejor definidos $2,4,9$. Para los pacientes con lesiones no resecables, la cirugía ofrece una buena paliación, con mejora de la calidad de vida, y se aso cia con un mínimo de morbilidad ${ }^{2-4}$. El papel de la cirugía, sin embargo, en el caso de las metástasis de melanoma al páncreas, es menos claro, ya que hay muy poca literatura disponible que demuestre la ventaja de supervivencia. En una serie, la supervivencia a los cinco años de los pacientes con metástasis múltiples mejoró del $23 \%$ al $37,5 \%$ con el tratamiento quirúrgico en pacientes seleccionados ${ }^{10,14}$. La mediana del periodo libre de enfermedad (PLE) en estos pacientes fue de 24 meses $^{13}$. En otra serie que involucró $\mathrm{a}$ cuatro pacientes, dos pacientes habían muerto por el seguimiento a los 25 meses y dos habían sobrevivido a los 30 y 76 meses $^{4}$. Se observó que los pacientes que sobrevivieron tuvieron un PLE de 4 y 14 años respectivamente Otros estudios, sin embargo, no muestran una mejora significativa de supervivencia después de la cirugía ${ }^{1,5,6,14}$. Parece que la supervivencia de los pacientes depende de dos factores principales: la capacidad de resecar completamente las metástasis, lo que hace que el paciente quede libre de la enfermedad y un PLE prolongado. Se cree que un aumento del PLE es resultado de una biología tumoral más favorable, donde las células tumorales son menos agresivas, lentas 
para dividirse y son menos propensas a metastatizar ${ }^{2}$. Desafortunadamente, actualmente no hay tratamiento efectivo no quirúrgico y el papel de la quimioterapia adyuvante y la inmunoterapia está siendo estudiada.

En conclusión, los estudios sugieren que, en un paciente con características tumorales favorables, que se pueden discernir por la duración del PLE y la resección completa de una lesión solitaria metastásica, la cirugía debe ser considerada como una opción viable de tratamiento, pero es necesario corroborar estos resultados con estudios más grandes.

\section{CONFLICTO DE INTERÉS}

Los autores declaran no tener conflictos de interés.

\section{BIBLIOGRAFÍA}

1. Goyal J, Lipson EJ, Rezaee N, et al. Surgical resection of malignant melanoma metastatic to the pancreas: case series and review of literature. J Gastrointest Cancer. 2012;43(3):431-6.

2. Nikfarjam M, Evans $P$, Christophi C. Pancreatic resection for metastatic melanoma. HPB (Oxford). 2003; 5(3):174-9.

3. Meyer T, Merkel S, Goehl J, Hohenberger W. Surgical therapy for distant metastases of malignant melanoma. Cancer. 2000; 89:1983-91.
4. Eidt S, Jergas M, Schmidt R, Siedek M. Metastasis to the pancreas-an indication for pancreatic resection? Langenbecks Arch Surg. 2007;392:539-42.

5. Crippa S, Angelini C, Mussi C, et al. Surgical treatment of metastatic tumors to the pancreas: a single center experience and review of the literature. World J Surg. 2006; 30:1536-42.

6. Hiotis SP, Klimstra DS, Conlon KC, Brennan MF. Results after pancreatic resection for metastatic lesions. Ann Surg Oncol. 2002;9:675-9.

7. Miao-Xia H, Bin S, Hui J, Xian-Gui H, Yi-Jie Z, Jian-Ming Z. Complete resection of isolated pancreatic metastatic melanoma: A case report and review of the literatura. World J Gastroenterol. 2010;16(36):4621-4.

8. Fletcher WS, Pommier RF, Lum S, Wilmarth TJ. Surgical treatment of metastatic melanoma. Am J Surg. 1998;175:413-7.

9. Reddy S, Edil BH, Cameron JL, et al. Pancreatic resection of isolated metastases from nonpancreatic primary cancers. Ann Surg Oncol. 2008;15:3199-206.

10. Wood TF, DiFronzo LA, Rose DM, et al. Does complete resection of melanoma metastatic to solid intra-abdominal organs improve survival? Ann Surg Oncol. 2001;8:658-62.

11. Lanitis S, Papaioannou N, Sgourakis G, Seitz A, Zacharakis E, Karaliotas C. Prolonged survival after the surgical management of a solitary malignant melanoma lesion within the pancreas: a case report of curative resection, J Gastrointestin Liver Dis. 2010;19(4):453-5.

12. Meyer T, Merkel S, Goehl J, Hohenberger W. Surgical therapy for dis tant metastases of malignant melanoma. Cancer. 2000;89:1983-91.

13. Lee CC, Faries MB, Wanek LA, Morton DL. Improved survival for stage IV melanoma from an unknown primary site. J Clin Oncol. 2009;27:3489-95.

14. Flaherty KT, Puzanov I, Kim KB, Ribas A, McArthur GA, Sosman JA, et al. Inhibition of mutated, activated BRAF in metastatic melanoma. NEJM. 2010;363:809-19. 